\title{
Sight related complaints after prolonged laparoscopic oncogynaecologic surgery
}

Grauslyte L., Zavackiene A., Baliuliene V., Macas A., Rimaitis K Lithuanian University of Health Sciences, Department of Anesthesiology,

Kaunas, Lithuania

\section{Introduction}

Minimally invasive surgery is used in order to reduce surgical aggression and to minimize morbidity. Recently the safety of prolonged laparoscopic surgery has been called into question due to the possibility of increase in intracranial pressure caused by pneumoperitoneum and Trendelenburg position ${ }^{1,2}$. One of the complications is thought to be related to sight.

\section{Goal and objectives}

$>$ To evaluate the rate of sight related complaints among women who underwent prolonged laparoscopic oncogynaecologic surgery.

$>$ To assess the impact of surgery duration on the incidence of complaints.

\section{Material and methods}

A retrospective cohort study was carried in a teaching hospital. Medical records were analyzed of women who underwent laparoscopic oncogynaecologic surgery lasting three hours or longer in the Trendelenburg position in 2013-2014. Subsequently all these women were contacted by phone in order to obtain the information regarding their sight following the surgery. Data was analyzed statistically, using Pearson's Chi squared test, significance level was set at $5 \%(p=0.05)$.

\section{Results}

Of 82 patients who fit the inclusion criteria, 57 (69.5\%) were successfully contacted and included in the study. Overall the average age of patients was $61.1( \pm 8.6)$ years. The average duration of surgery reached 217.8 ( \pm 58.2 ) minutes, ranging from 3 to 8 hours. The complaints related to sight that patients expressed following the surgery are supplied in Table 1 . The rate of those complaints statistically significantly depended on the duration of surgery $(p=0.011)$. The incidence of sight related complaints depending on the duration of surgery is supplied in Figure 1.
Table 1. Incidence of sight related complaints among all study participants $(n=57)$

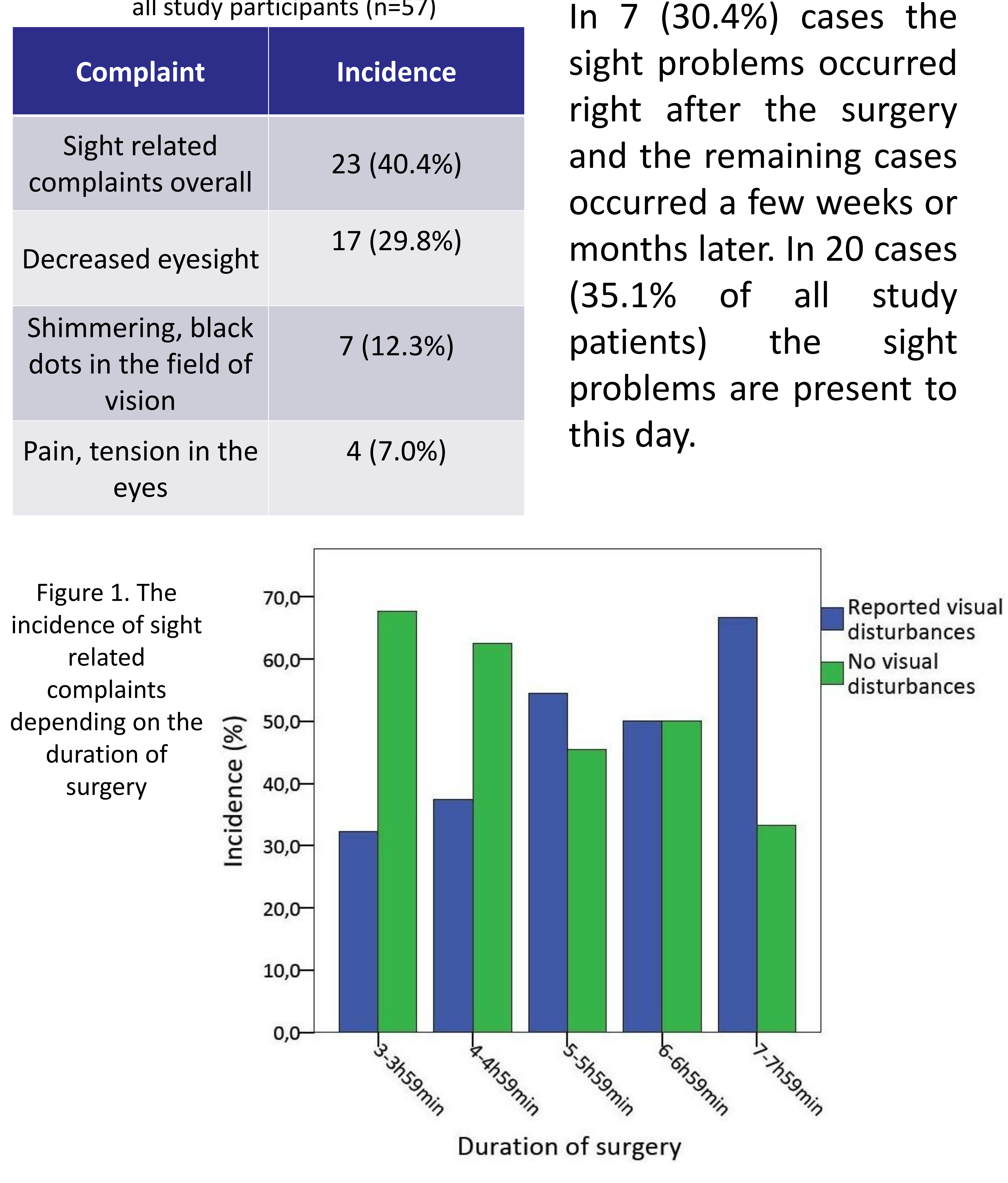

\section{Conclusions}

$>$ The rate of sight related complaints among patients following prolonged laparoscopic surgery is prominent and correlates to the duration of surgery.

$>$ It shows that patient-safety oriented guidelines for prolonged laparoscopic surgery need to be created, proving the necessity of future research aimed at evaluating the direct link between changes in intracranial pressure and prolonged laparoscopic surgeries.

\section{References}

1 Nuzzi R, Tridico F. Ocular complications in laparoscopic surgery: review of existing literature and possible prevention and treatment. Semin Ophthalmol 2015 Aug 19:1-9

2 Taketani Y, Mayma C, Suzuki N, Wada A, Oka T, Inamochi K, Nomoto Y. Transient but significant visual field defect after robot-assisted laparoscopic radical prostatectomy in deep Trendelenburg position. PLoS One 2015 Apr 23;10(4):e0123361. 